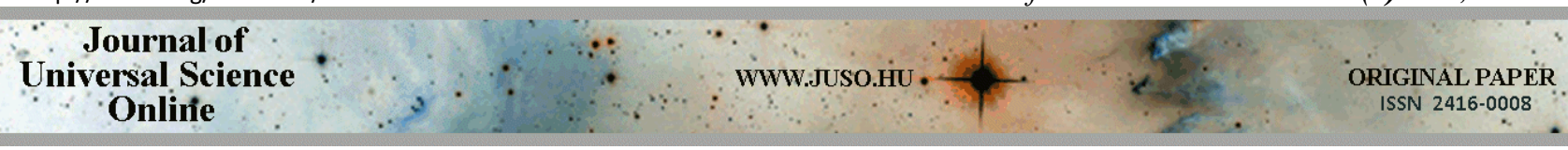

\title{
Rapid BRAF mutation detection in melanoma patients by immunohistochemistry
}

\section{László FÜLÖP ${ }^{1}$, Katalin GÖTZER ${ }^{1}$, Erzsébet CSERNÁK ${ }^{1}$, Danyil Szergejevics KUZNYECOV ${ }^{1}$ and Erika TÓTH ${ }^{*}$}

\author{
${ }^{1}$ National Institute of Oncology, Surgical and Molecular Pathology Center, Ráth Gy. u. 7-9, Budapest, H-1122 Hungary \\ *Corresponding Author; e-mail: erika66toth@gmail.com
}

Received: 11 March 2017 / Revised: 3 April 2017 / Accepted: 29 April 2017

Keywords: melanoma, BRAFVE1, immunohistochemistry

\begin{abstract}
The V600E mutation is the most common ( $90 \%)$ activating mutation of the BRAF gene. $B R A F$ mutations have been frequently investigated in melanoma, colorectal cancer and papillary thyroid carcinoma. The importance of the detection of $B R A F$ mutations has been rising by the routine use of Braf inhibitor therapy.

We evaluated the usefulness of the $B R A F$ V600E mutation-specific monoclonal antibody (VE1) in metastatic melanoma patients. To confirm the results of immunohistochemistry (IHC), we used COBAS $4800 B R A F$ V600 mutation test and PCR amplification followed by Sanger sequencing. 36 of 105 patients have wild-type $B R A F$ gene, 64 have V600E mutation and 5 of 105 have V600K mutation. Predicting the mutation only by IHC using VE1 antibody, all 58 positively scored specimen were V600E mutant. The V600K, the wild-type patients and 7 patients from the V600E mutant group scored as negative. Thus the specificity is $100 \%$ and the positive predictive value is 1 of the IHC method. After processing our data we could establish a cheaper diagnostic algorithm for rapid detection of $B R A F$ mutation.
\end{abstract}

\section{Introduction}

Approximately 30 connections of the $B R A F$ gene with human cancers have been identified. (1-3\% non-small-cell lung cancer [1], 5\% in colorectal cancer papillary thyroid carcinoma [2], 57\% of Langerhans cell histiocytosis, $100 \%$ of hairy cell leukaemia [3], ameloblastoma, papillary craniopharyngioma, high grade gliomas in children [4], Metanephric adenoma [5, 6].

Hyperactivation of growth signal is usually a key factor in the development of these malignomas. Beside the mutations affecting the first steps of the signal (GFR and Ras) about $60 \%$ of the melanomas carrying an activational mutation in the BRAF gene [6]. In $90 \%$ of the mutant cases, thymine is substituted with adenine at nucleotide 1799. This leads to valine $(\mathrm{V})$ being substituted for by glutamate $(\mathrm{E})$ at codon 600 (now referred to as V600E mutation).

In metastatic melanoma patients B-Raf inhibitor therapy is accepted in 2011 [7], and more selective inhibitors approved by FDA in 2013 [8]. Braf inhibitor treatment is suggested to be applied even beyond progression $[7,9]$.

Detection of the presence of BRAF mutation is the requirement of treatment and so in the given cases fast and reliable 


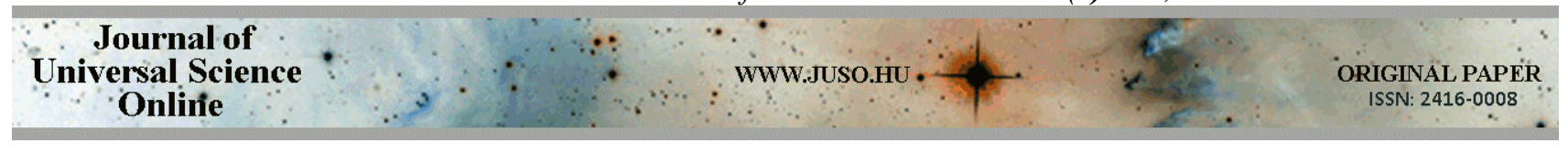

mutation detection is very important. VE1 antibody recognises only V600E mutant Braf but not the wild form or other mutant variant [10]. Despite of the works demonstrating the relevance of the Braf $\mathrm{V} 600 \mathrm{E}$ mutation specific antibody VE1 [11, 12, 13, 14, 15] it is still not accepted in the everyday routin.

\section{Material and methods}

Formalin fixed paraffin embedded (FFPE) blocks of 104 metastatic melanoma patients were analyzed. Anti-BRAFV600E staining was performed on the same blocks used for molecular analysis, using the VE1 mouse monoclonal antibody (Ventana Medical Systems, Roche, CN: 790-4855) diluted 1/100.
Despite of the few analitical difficulties here we show a diagnostic procedure in wich immunohistochemistry has a reliable role.

The importance of the VE1 antibody is highlighted by the faster diagnosis and lower procedure costs.

\section{Results}

\section{BRAF mutation analysis}

Cobas ${ }^{\circledR} 4800$ BRAF V600 Mutation Test was performed according to manufacturer instructions (Supplementary material 1).

In house method were also used. PCR amplification with mutation specific primer followed by Sanger sequencing was performed as described before [16].

We examined 104 patients with metastatic melanoma. In 50 cases analysis were made from the metastasis, and the rest of the cases were primary melanomas.

Table 1. Discordant cases in detail

\begin{tabular}{|c|c|c|c|c|c|c|}
\hline $\begin{array}{c}\text { ID } \\
\text { Number }\end{array}$ & $\mathrm{IHC}$ & $\begin{array}{c}\text { COBA } \\
\mathrm{S}\end{array}$ & Sanger & $\begin{array}{l}\text { percentage } \\
\text { of tumor cells }\end{array}$ & Origin & $\begin{array}{c}\text { Probable cause of negativ } \\
\text { IHC }\end{array}$ \\
\hline 10 & negative & V600 & V600E & $80 \%$ & primer tumor & low positivity \\
\hline 31 & negative & V600 & V600E & $90 \%$ & metastasis & low positivity \\
\hline 35 & negative & V600 & V600E & $90 \%$ & primer tumor & low positivity \\
\hline 37 & negative & V600 & V600E & $80 \%$ & metastasis & low positivity \\
\hline 40 & negative & V600 & V600E & $70 \%$ & primer tumor & low positivity \\
\hline 41 & negative & V600 & V600K & $80 \%$ & primer tumor & V600K \\
\hline 64 & negative & V600 & V600E & $90 \%$ & metastasis & $\begin{array}{l}\text { low positivity } \\
\text { high staining noise }\end{array}$ \\
\hline 72 & negative & V600 & V600E & $90 \%$ & metastasis & high staining noise \\
\hline 82 & negative & V600 & V600K & $80 \%$ & primer tumor & V600K \\
\hline 83 & negative & V600 & V600K & $80 \%$ & primer tumor & V600K \\
\hline 84 & negative & V600 & V600K & $>5 \%$ & metastasis & V600K \\
\hline 85 & negative & V600 & V600K & $80 \%$ & primer tumor & V600K \\
\hline 92 & negative & V600 & V600E & $80 \%$ & metastasis & low positivity \\
\hline
\end{tabular}

We performed Braf IHC and COBAS 4800 V600 mutation test on all specimens, and Sanger sequencing on the discordant cases. Regarding these discordant cases COBAS and Sanger sequencing were 100\% congruent. 48 patients were counted as negative and 56 as positive with Braf IHC. All the positively scored patients turned out to be BRAF mutants with COBAS. Five specimens of the 48 IHC negatively scored group had $\mathrm{V} 600 \mathrm{~K}$ mutation and 8 happened to be false negative (Figure 1). Detailed 


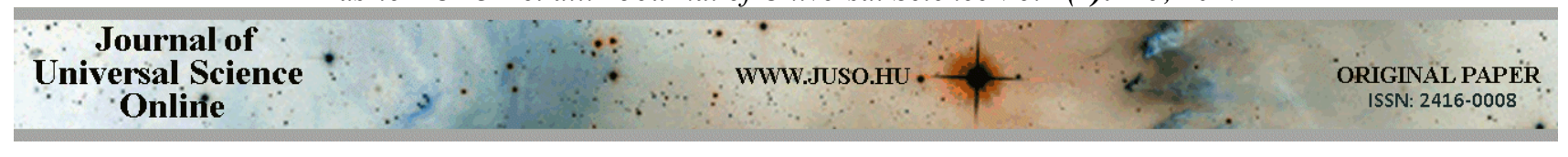

information of the discordant cases are shown in Table 1. Representative pictures are shown in Figure 2. Age of the BRAF mutation carrying patients were significantly lower (average: 58.42 years and 66.13 years resp., $\mathrm{p}=0.005)$.

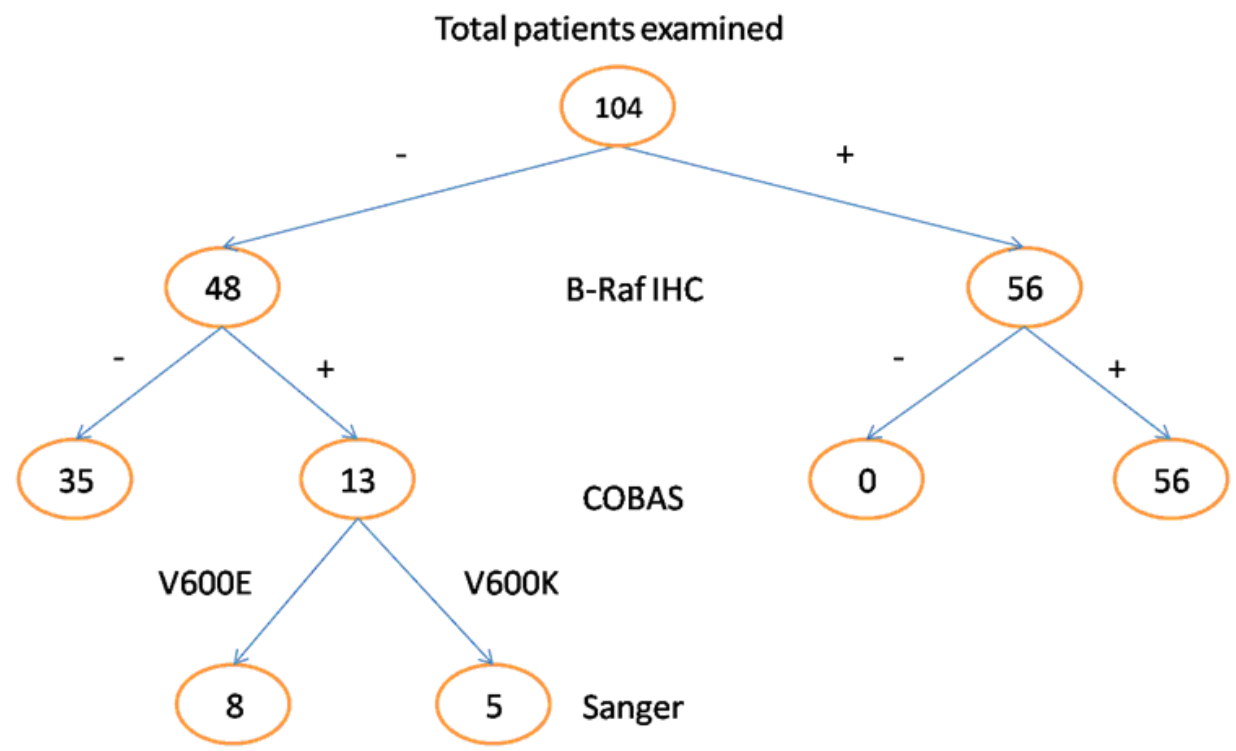

Fig. 1. Analisis sequence. IHC: immunohistochemistry

\section{Discussions}

In this study we further asserted the role of Braf VE1 immunohistochemistry in the diagnosis of BRAF mutations. Foremost we verified BRAF mutation status by COBAS or in house method. Discordant cases were analysed by Sanger sequencing, revealing V600K mutations in the majority of false negative cases. Since intra-observer variability was negligible the false negative cases were re-analysed.

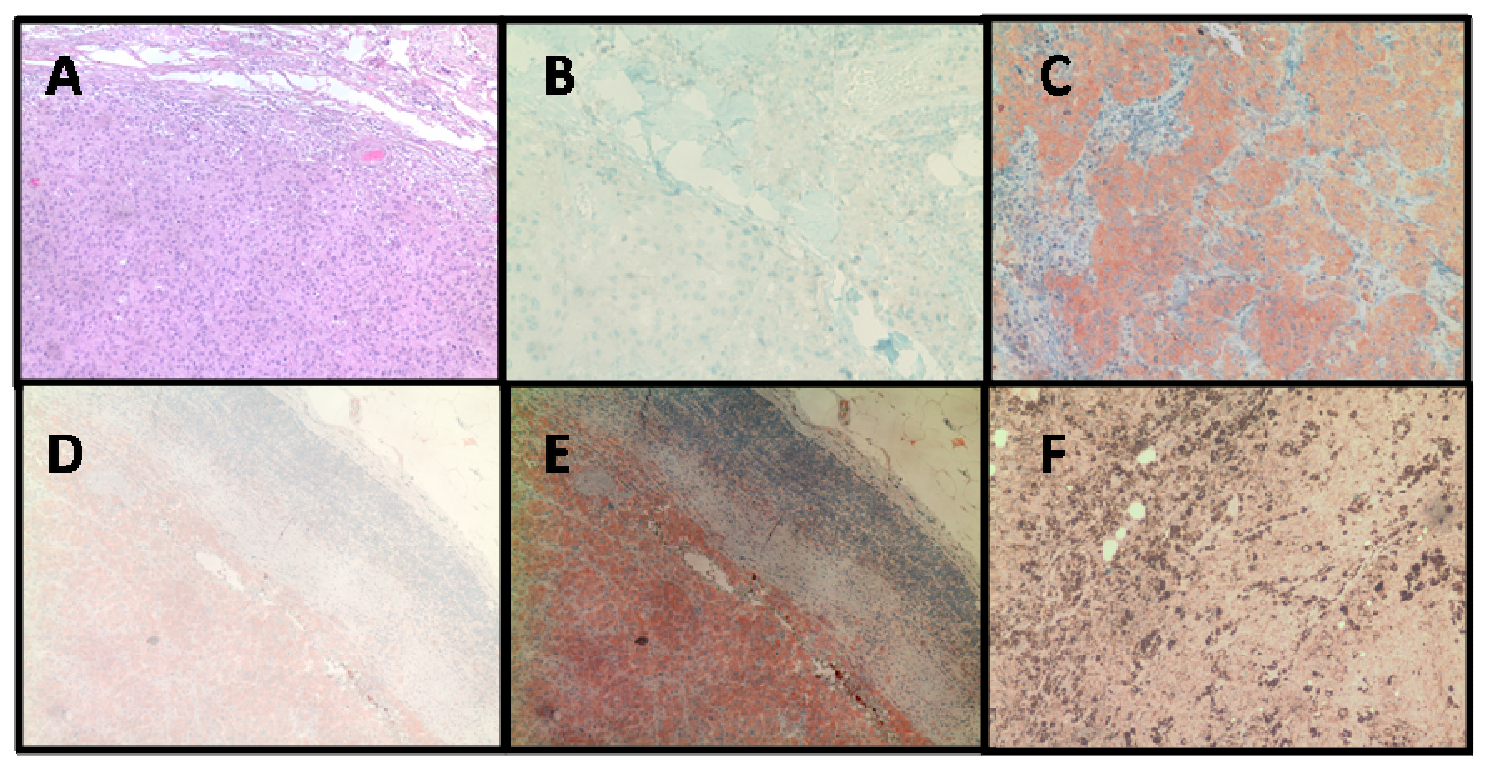

Fig. 2. Representative pictures. A: standard hematoxilin-Eosin staining. B: True negative (negative IHC, wild type). C: True positive (positive IHC, V600 mutant). D: False negative (negative IHC, V600K mutant) E: False nagative after image processing. F: Highly pigmented area, counted as negative. 


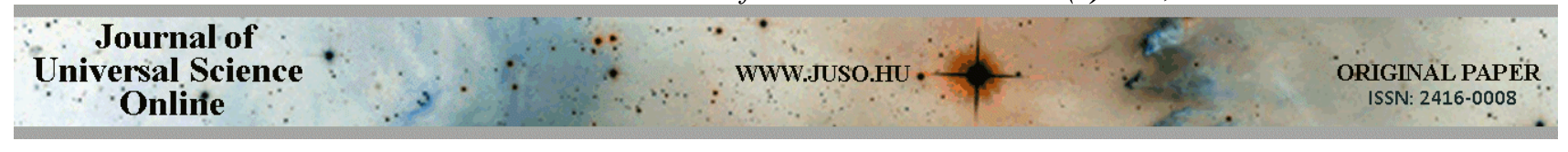

This showed that true negative cases were reliably negative, but false negative cases had usually problematic IHC due to the highly pigmented areas, high background

Braf VE1 IHC is an easy method that doesn't require DNA isolation and can be performed during short time. Positive immuno-histochemistry means the presence of BRAF mutation [11, 12, 13]. But we cannot rely on negative or uncertain Braf VE1 IHC, because of the V600K mutants and regarding the therapeutic consequences we shall be completely sure about the mutation.

\section{References}

1. Sasaki H, Maekawa M, Tatematsu T, et al. (2015) Increased BRAF copy number in lung adenocarcinoma. Oncol Lett 9:709-712. doi: 10.3892/ol.2014.2719

2. Lu J, Gao J, Zhang J, et al. (2015) Association between BRAF V600E mutation and regional lymph node metastasis in papillary thyroid carcinoma. Int J Clin Exp Pathol 8:793-9.

3. Arora N, Nair S, Pai R, et al. V-raf murine sarcoma viral oncogene homolog B (BRAF) mutations in hairy cell leukaemia. Indian J

Pathol Microbiol 58:62-5. doi: 10.4103/0377-4929.151190

4. Mistry M, Zhukova N, Merico D, et al. (2015) BRAF Mutation and CDKN2A Deletion Define a Clinically Distinct Subgroup of Childhood Secondary High-Grade Glioma.

J Clin Oncol 33:1015-22. doi: 10.1200/JCO.2014.58.3922

5. Udager AM, Pan J, Magers MJ, et al. (2015)

Molecular and Immunohistochemical Characterization Reveals Novel BRAF

Mutations in Metanephric Adenoma.

Am J Surg Pathol 39:549-57. doi: 10.1097/PAS.0000000000000377

6. Davies H, Bignell GR, Cox C, et al. (2002) Mutations of the BRAF gene in human cancer. Nature 417:949-54. doi: 10.1038/nature00766 noise or very low positivity. These can highlight potential positive cases but still recommended to count as negative and perform DNA tests.

So we strongly suggest BRAFVE1 immunohisto-chemistry as a primary screening method and to evaluate every negative or uncertain cases by further molecular methods.

Pre-screening melanoma with Braf VE1 IHC can shorten the time for the appropriate therapy in the majority of cases, due to the excellent positive predictive value.

7. Chapman PB, Hauschild A, Robert C, et al. (2011) Improved survival with vemurafenib in melanoma with BRAF V600E mutation.

N Engl J Med 364:2507-16. doi: 10.1056/NEJMoa1 103782

8. Thompson CA (2013) FDA approves two new drugs against advanced melanoma. Am J Health Syst Pharm 70:1094. doi: $10.2146 /$ news 130045

9. Scholtens A, Geukes Foppen MH, Blank CU, et al. (2015) Vemurafenib for BRAF V600 mutated advanced melanoma: results of treatment beyond progression. Eur J Cancer 51:642-52. doi: 10.1016/j.ejca.2015.01.009

10. Capper D, Preusser M, Habel A, et al. (2011) Assessment of BRAF V600E mutation status by immunohistochemistry with a mutationspecific monoclonal antibody.

Acta Neuropathol 122:11-9. doi: 10.1007/s00401-011-0841-z

11. Liu H, Li Z, Wang Y, et al. (2014) Immunohistochemical detection of the BRAF V600E mutation in melanoma patients with monoclonal antibody VE1.

Pathol Int 64:601-6. doi: 10.1111/pin.12215

12. Løes IM, Immervoll H, Angelsen J-H, et al. (2014) Performance comparison of three BRAF V600E detection methods in malignant 


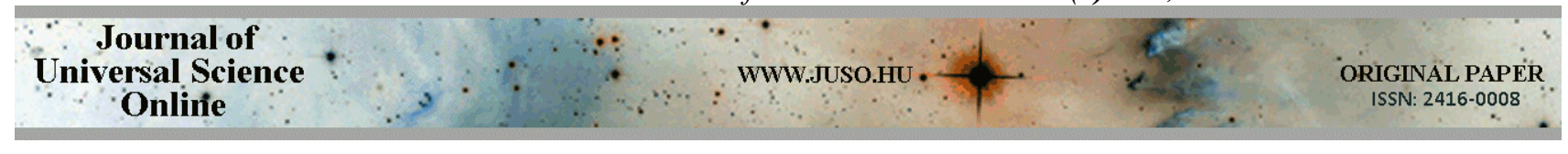

melanoma and colorectal cancer specimens.

Tumor Biol 36:1003-1013.

doi: 10.1007/s13277-014-2711-5

13. Pearlstein M V, Zedek DC, Ollila DW, et al. (2014) Validation of the VE1 immunostain for the BRAF V600E mutation in melanoma.

J Cutan Pathol 41:724-32.

doi: $10.1111 /$ cup. 12364

14. Tetzlaff MT, Pattanaprichakul P, Wargo J, et al. (2015) Utility of BRAF V600E

Immunohistochemistry Expression Pattern as a Surrogate of BRAF Mutation Status in 154
Patients with Advanced Melanoma.

Hum Pathol 46:1101-1110.

doi: 10.1016/j.humpath.2015.04.012

15. Manfredi L, Meyer N, Tournier E, et al. (2015) Highly Concordant Results Between Immunohistochemistry and Molecular Testing of Mutated V600E BRAF in Primary and Metastatic Melanoma. Acta Derm Venereol. doi: $10.2340 / 00015555-2326$

16. Sanger F, Nicklen S, Coulson AR (1977) DNA sequencing with chain-terminating inhibitors.

Proc Natl Acad Sci U S A 74:5463-7. 